\section{EVALUATING PARTICIPATION IN CHILDREN WITH CEREBRAL PALSY}

S.I. Michelsen ${ }^{1}$, E.M. Flachs ${ }^{1}$, P. Due ${ }^{1}$, P. Uldall ${ }^{2}$

${ }^{1}$ National Institute of Public Health, University of Southern Denmark, ${ }^{2}$ The Juliane Marie Centre, Rigshospitalet, Copenhagen University Hospital, Copenhagen, Denmark

Background and aims: The European SPARCLE study of children with cerebral palsy responds to a growing interest in evaluating children with disabilities according to their quality of life and participation in life situations. The aim of this presentation is to report for the Danish children in the SPARCLE study 1) participation and physical, social and attitudinal environment 2) their parent's view of the social security system and 3 ) international differences in participation and the environment needed for children with cerebral palsy.

Methods: There were 115 Danish children with cerebral palsy age 8 to 12 years and their families (818 children in the SPARCLE study). Measures used were Life-H, Frequency of Participation Questionnaire and the European Child Environment Questionnaire.

Results: Danish children with cerebral palsy aged 8 to 12 had a high level of participation in everyday life and participated, for example, as often in sport and outdoor games as Danish children in the general population. The environment often fulfilled the needs of these children. Danish children with cerebral palsy had much higher participation, compared with children with cerebral palsy in other European regions. Denmark was among the European regions providing children with cerebral palsy with the most accessible environment.

Conclusions: The high level of participation of Danish children demands much human assistance, aids and adaptations. It is likely that this human assistance is mainly provided by parents. Danish parents call for better information regarding social support and we need more information regarding well-being of parents of children with cerebral palsy.
40

PARENTAL COPING AND RESOURCES WHEN THE CHILD IS SEVERELY DISABLED - HOW TO INFORM AND SUPPORT THE PARENTS?

A.H. Graungaard ${ }^{1}$, J.S. Andersen ${ }^{1}$, L. Skov ${ }^{2}$

${ }^{1}$ Department of General Practice, University

of Copenhagen, Copenhagen, ${ }^{2}$ Department of

Paediatrics, Glostrup University Hospital, Glostrup, Denmark

Background and aims: Parents who realise that their newborn child is severely disabled often experience severe physical and emotional stress. Parental well-being is essential for the care-taking of the child. It is yet not known why some cope well and others do not. The aim of this study was to explore how parents coped with parenting a disabled child, maintained their energy and personal resources, and how health professionals could support this processes.

Methods: We conducted a qualitative, longitudinal interview study with 16 parents during the first two years after their child was diagnosed with a severely disabling condition. A grounded theory analysis was conducted and findings were interpreted in a theoretical framework of Lazarus and Folkman's studies on coping and Fredrickson's broaden-andbuild theory of positive emotions, as well as theories of positive illusions and benefit finding during severe adversity.

Results: We found that parents continually created and sustained their personal resources through positive reappraisals of their circumstances. Nine general coping strategies were identified as; emotional bonding, identifying supportive social relations, creating mental respite, meaning-making, benefit finding, evaluating existential beliefs, taking action, normalization and maintaining hope. A theory of resource-creation is proposed as an addition to the current understanding of coping and the role of positive emotions.

Conclusions: We found that parental coping was directed at creating new resources in the family as well as solve practical problems and reduce emotional strain. Implications for information and counselling are provided. 